\title{
Les Opérations des Compagnies d'Assurance IARD : Identification de Modèles et Simulation d'Hypothèses de Conjoncture Economique
}

\author{
par J.-François Outreville *
}

\section{Introduction}

L'analyse prévisionnelle des états comptables est un domaine de recherche relativement récent et en pleine évolution aussi bien en finance qu'en comptabilité ${ }^{1}$. Il a même été démontré 2 que la variabilité des résultats des entreprises pouvait être expliquée par des variables macroéconomiques, en d'autres termes attribuable en partie à la conjoncture économique. Or la modélisation macroéconomique dans le secteur des assurances n'a jusqu'à présent pas eu la part qu'elle serait en droit d'avoir. Aux Etats-Unis, l'analyse la plus complète est celle de J. David Cummins [1975] et concerne le secteur de l'assurance-vie. Aucune étude semblable n'a été développée pour le secteur des assurances générales.

Plus récemment, en France, la modélisation macroéconomique du secteur des assurances a fait l'objet de recherches aussi bien pour le secteur de l'assurance-vie que pour celui des assurances I.A.R.D. ${ }^{3}$.

Le but d'une telle analyse est d'explorer les liens entre le secteur des assurances et les autres secteurs de l'économie principalement mesurés par l'impact de variables macroéconomiques sur le comportement du secteur. Une utilisation intéressante de cette approche réside dans son application à un compte d'exploitation prévisionnel pour l'entreprise. Les limites sont celles de l'analyse macroéconomique et de l'utilisation d'agrégats qui ne reflètent que le comportement moyen et non le comportement d'un individu ou d'une firme en particulier. Il est certain, pourtant, que cette approche

* Professeur, Faculté des Sciences de l'Administration, Université Laval, Québec. Je tiens à remercier Yves Roy, ainsi que deux "referees » de la revue pour leurs commentaires et propositions. Il est évident que je reste seul responsable des possibles erreurs et omissions.

1 Nous faisons référence aux travaux de Ball and Brown [1968], Ball and Watts [1972], Beaver [1970], Brooks and Buckmaster [1976] et Lookabill [1970].

2 Brown and Ball [1967].

3 J. F. Outreville [1979]. 
devrait être aujourd'hui considérée par les entreprises d'assurance 4 . L'orientation de cette étude est principalement empirique et tend à montrer l'impact de variables macroéconomiques sur les opérations des compagnies d'assurance-dommages. Après avoir présenté le cadre conceptuel de l'étude, nous montrerons qu'il est possible d'envisager des relations statistiques satisfaisantes lorsque l'on fait intervenir principalement la variable "inflation », et que ces modèles sont statistiquement plus valides que de simples relations linéaires, exponentielles ou autoregressives. Enfin dans la dernière partie, nous proposerons une application de simulation d'hypothèses de conjoncture économique aux opérations de compagnies d'assurance-dommages.

\section{Le cadre conceptuel}

\subsection{Les variables $d u$ secteur}

Afin de réaliser l'équilibre de ses comptes, une société a deux types de ressources, les primes et accessoires $(Q)$ d'une part, les ressources dues à ses placements $(P L)$ d'autre part. En d'autres termes, ces deux ressources sont celles du service production et du service financier.

Afin d'intégrer dans une même analyse le compte d'exploitation et le compte général des pertes et profits, le concept d'accroissement des provisions techniques $(P T)$ est étendu à l'ensemble des provisions et réserves de la société et le concept de produit financier $(F)$ étendu en intégrant les plus-values et moins-values sur cessions d'éléments d'actifs.

Afin de décrire le processus économique et le fonctionnement des comptes de la société, nous présentons un schéma ${ }^{5}$ où les deux types de ressources sont représentés. Il permet de résumer la réalisation de l'équilibre des comptes de la société et d'introduire les concepts macroéconomiques de revenu, prix et rendement.

Dans notre schéma de détermination des provisions et placements, nous allons analyser les différents flux qui permettent, à partir des ressources de production $(Q)$ et financières $(F)$, de constituer les placements $(P L)$ dans le respect des égalités comptables. Outre la prime pure $(Q)$ destinée à la couverture du risque assuré, l'assureur doit percevoir une majoration destinée :

- à rémunérer l'intermédiaire qui apporte l'affaire, la commission $(\mathrm{Co})$;

- à couvrir ses frais généraux, les charges $(\mathrm{Ca})$;

- à constituer une réserve de sécurité si le risque est susceptible de subir d'une année à l'autre, des fluctuations importantes, ou si le risque est insuffisamment connu ;

- à lui permettre d'espérer un bénéfice raisonnable dont une part peut être ristournée aux assurés non sinistrés au cours de l'année (participation aux bénéfices).

4 Recommandation de l'Institut Canadien des Actuaires à ses membres lors du dernier congrès annuel, d'envisager l'utilisation de modèles prévisionnels économétriques (Globe and Mail, Friday Nov. 14, 1980).

5 Ce schéma est inspiré d'une étude de H. Bohman [1979]. 


\subsection{Les égalités comptables du secteur}

Pour un exercice comptable donné, il existera une relation du type :

$$
Q \gtrless C o+C a+S+\Delta P T,
$$

où $S$ est le montant des sinistres effectivement payés par le secteur ; $\triangle P T$ la variation des provisions techniques déterminée par la relation suivante :

$$
\triangle P T=P T_{t}-P T_{t-1} \text {. }
$$

Si pour un exercice donné le terme de gauche de la relation (1) est supérieur au terme de droite, il se dégagera alors un «profit pur» de souscription $W_{1}$. Ce profit, que l'on peut appeler aussi de production, est différent du bénéfice éventuel dégagé au compte d'exploitation des sociétés, puisque ce compte inclut le (produit-charge) des placements.

Si le sens de la relation est inverse, l'apport du produit financier dans une proportion $j$ sera nécessaire pour réaliser l'égalité comptable :

$$
Q+j . F=S+C o+C a+\Delta P T \text {. }
$$

Mais si :

$$
Q+j . F<S+C o+C a+\Delta P T,
$$

l'ensemble des ressources financières sera insuffisant pour réaliser l'égalité comptable et il subsistera une perte d'exploitation $\left(-W_{2}\right)$.

Si nous continuons notre schématisation (exception faite du problème des impôts et taxes), le flux d'exploitation $W_{2}$ viendra accroître (ou décroître : il existe une réserve complémentaire obligatoire) l'ensemble des fonds propres et réserves $C P$. On peut appliquer à $C P$ le même raisonnement qu'aux placements, à savoir qu'avec un certain taux d'inflation annuel $i$, on devrait avoir :

$$
C P_{t}=(1+i) C P_{t-1},
$$

pour maintenir les capitaux propres et réserves au même niveau en monnaie courante. On aura donc un flux $k . F$ nécessaire à la réalisation de l'égalité comptable. Soit $R a$ le taux de rentabilité des placements, on aura alors $F=\operatorname{Ra} . P L$ et la relation suivante :

$$
R a . P L \gtreqless F(j+k) \text {. }
$$

A nouveau si le terme de gauche de cette relation est supérieur au terme de droite, il subsistera alors un «profit pur » financier $W_{3}$ qui viendra accroître (déduction faite des distributions éventuelles de bénéfices et participations) l'ensemble des fonds propres.

L'ensemble de la démarche précédente est résumé dans le graphique 1 de schématisation des comptes.

D'autre part, l'analyse purement descriptive que nous venons de formuler correspond bien à l'analyse théorique de la « situation de risque » d'une entreprise d'assu- 
rance telle que l'on peut la trouver décrite par K. Borch ([1974], p. 88). Si l'on considère une entreprise en faisant l'hypothèse que toutes les primes concernant les contrats en cours ont été encaissées et que tous ces contrats expirent à la fin d'une même période $(t, t+1)$, la « situation de risque » de l'entreprise sera déterminée par deux éléments : la probabilité de sinistralité et la somme des fonds collectés par l'entreprise pour la couverture de ces sinistres.

\subsection{Les relations linéaires et ratios du secteur}

A) Commissions et charges

Soit $Q$ la prime commerciale et $c$ le ratio de chargement, c'est-à-dire la couverture des commissions et charges d'exploitation par la prime commerciale, la prime pure $\bar{Q}$ sera égale à :

(7)

$$
\begin{aligned}
& \bar{Q}=Q-c Q, \\
& \text { où } c=\frac{C o+C a}{Q} .
\end{aligned}
$$

Les commissions et charges sont aussi définies habituellement comme une fonction proportionnelle de la prime telle que (Depoid [1967]) :

$$
\begin{aligned}
& C o=\gamma \cdot Q \\
& \text { (9) } C a=a+\beta Q \text {, }
\end{aligned}
$$

$\beta$ correspondant à la gestion des sinistres et $\alpha$ étant une constante relative aux frais de quittancement.

B) La sinistralité

On définit habituellement le ratio de sinistralité en fonction des primes :

$$
s=\frac{S}{Q} .
$$

L'accroissement de la sinistralité étant un facteur exogène pour les sociétés d'assurance, ce ratio permet de savoir dans quelle mesure les sociétés parviennent à une certaine stabilité par compensation soit sur la cadence des règlements de sinistres, soit sur l'encaissement des primes. En réalité, il y a peu de chance que ce ratio soit très stable dans le temps et s'il existe une relation linéaire stable, c'est plutôt celle liant le niveau de sinistralité à celui des provisions techniques.

C) Les provisions techniques

Dans sa théorie collective du risque, F. Lundberg [1909] propose de définir les réserves $(P T)$ d'une entreprise d'assurance à un moment $t$ par la relation :

$$
P T_{t}=P T_{o}+\bar{Q}_{t},
$$

où $\bar{Q}_{t}$ est le total des primes pures encaissées par la compagnie pendant la période $(o, t)$. 


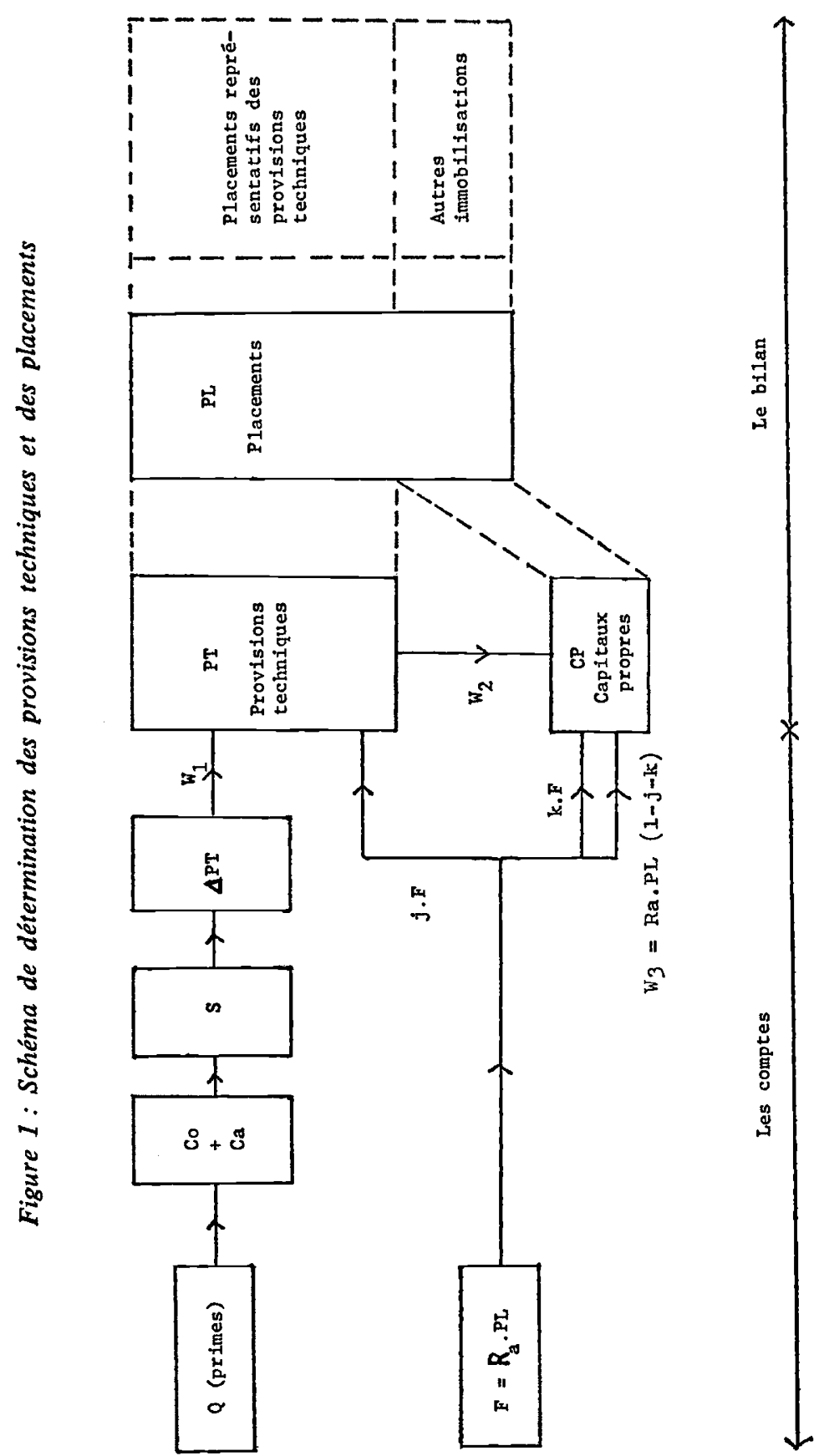


Pour un exercice donné, l'accroissement des provisions techniques de la société dépendra, d'après le schéma décrit au paragraphe précédent, de la sinistralité $(S)$ et du flux de ressources financières et d'exploitation ${ }^{6}$. Si $P T_{t-1}$ est le montant des provisions techniques au début de l'exercice, $\bar{Q}_{t}$ le montant des primes de l'exercice, on devrait avoir à la fin de l'exercice, dans le cas où la hausse des prix $i$ n'est pas prise en compte au niveau de la prime 7 , un niveau de provisions techniques optimal :

$$
P T^{*}{ }_{t}=(1+i) P T_{t-1}+a \cdot \bar{Q}_{t}-S_{t},
$$

le facteur $(1+i)$ permettant d'ajuster le niveau des provisions au taux d'inflation annuel ; le facteur $(a)$ permettant d'ajuster le niveau des provisions à la production effectivement réalisée pendant l'exercice; $S_{t}$ est le montant de sinistres effectivement payé au cours de l'exercice.

Si l'on admet que les provisions techniques suivent un processus d'ajustement de stock de type :

$$
P T_{t}-P T_{t-1}=\Theta\left(P T_{t}^{*}-P T_{t-1}\right),
$$

et s'il exjiste bien une relation stable entre sinistralité et provisions techniques :

$$
S_{t}=\alpha+\beta P T_{t-1},
$$

$\alpha$ et $\beta$ représentant respectivement la part non prévue et prévue des sinistres, alors la relation décrivant l'accroissement de ces mêmes provisions techniques sera :

$$
\Delta P T_{t}=-\Theta \alpha+\Theta \cdot(i-\beta) P T_{t-1}+\Theta \cdot a \bar{Q}_{t} .
$$

\section{Analyse économétrique et résultats}

\subsection{Méthodologie}

Le but de cette section étant de vérifier, ou non, l'existence de relations stables sur la période d'étude, la vérification empirique que nous présentons ici reprend les équations du paragraphe précédent. Afin de démontrer l'intérêt de cette approche, nous comparerons nos résultats à ceux de deux autres types de modèles:

- un modèle de croissance linéaire:

$$
\hat{Y}_{t}=a+b t
$$

6 Le modèle développé par Lundberg partait de l'hypothèse que les compagnies ne versent pas de dividende. De Finetti [1957] a montré qu'avec une telle hypothèse, on arrivait à des provisions techniques (réserves) infinies et a fait l'hypothèse d'une limite supérieure qui implique la distribution de dividendes sur les bénéfices. Karl Borch, quant à lui, a généralisé le modèle en introduisant le problème de la réassurance, problème que nous avons volontairement ignoré afin de simplifier notre analyse, mais surtout parce que nous raisonnons au niveau global.

7 Cette hypothèse n'est pas irréaliste puisqu'en raison du caractère annuel de la prime, de la nécessité d'obtenir l'accord de l'assuré et des pouvoirs publics (assurance I.A.R.D.) pour la révision, il faut au minimum un an pour qu'une augmentation produise tous ses effets au niveau de la prime. 
où $t$ est la seule variable indépendante « explicative » dénotant le déroulement du temps ;

- un modèle autoregressif :

$$
\hat{Y}_{t}=a+b Y_{t-1}
$$

Nous nous intéresserons particulièrement à l'impact de l'inflation sur la stabilité des comptes des sociétés. Alors que la demande d'assurance et les placements des sociétés sont influencés par les anticipations des décideurs face à l'inflation ${ }^{8}$, les éléments comptables que nous considérons ici (paiement des sinistres, frais de gestion et d'exploitation) sont eux affectés directement par la hausse des prix présente ou passée. Pour mieux en connaître l'incidence, nous avons choisi d'utiliser la procédure d'Almon ${ }^{9}$ et la méthode des simples moindres carrés sans avoir recours à la procédure de correction de l'autocorrélation des résidus, afin d'utiliser le test de Durbin-Watson comme mesure de la bonne spécification ou non des relations testées.

L'ensemble des relations ont été testées sur la période 1955-1975. Les rapports au Président de la République sur l'activité des organismes d'assurance et de capitalisation nous ont permis de calculer les variables du secteur des assurances utilisées dans cette étude. Les agrégats économiques, principalement le taux d'inflation calculé à partir de l'indice des prix à la consommation (PRIX), sont extraits du Bulletin mensuel de Statistique de l'INSEE.

\subsection{Résultats}

A) Les charges d'exploitation

Nous présentons successivement les résultats des relations en termes de ratio (charges / primes) et de montants (en millions de francs).

$\begin{array}{lcc}\text { ratio } & \bar{R}^{2} & D . W . \\ \text { modèle de croissance linéaire } & 0.838 & 0.394 \\ \text { modèle autoregressif } & 0.942 & 1.276 \\ \text { modèle proposé } & 0.975 & 1.840 \\ c=0.3551-0.0053 T+\sum_{i=0}^{7} \operatorname{lag}_{i} \text { PRIX } & \\ \quad[-18.168] \text { ing } & \\ \text { entre [ ] test de Student } & & \end{array}$

8 Voir à ce sujet J. F. Outreville ([1979], chap. 2 et 3).

9 J. J. Thomas [1978] présente clairement les problèmes que pose l'utilisation de la procédure des «lags d'Almon». La plupart des études utilisant cette technique se basent sur l'estimation qui maximise $R^{2}$ ou minimise l'erreur standard de la regression (S.E.). Nous estimons, et nous rejoignons en cela J. J. Thomas, qu'il est préférable de maximiser le couple $\left(R^{2}, D W\right)$ où $D W$ est le test de Durbin-Watson utilisé comme indicateur de la bonne spécification du modèle. 
Polynôme du $3^{\mathrm{e}}$ degré sans contraintes :

$$
\begin{array}{cccc}
\text { Lag } & 0 & 0.00048 & {[1.069]} \\
1 & 0.00171 & {[8.184]} \\
2 & 0.00218 & {[7.217]} \\
3 & 0.00209 & {[7.275]} \\
4 & 0.00167 & {[7.467]} \\
5 & 0.00114 & {[6.022]} \\
6 & 0.00072 & {[4.246]} \\
7 & 0.00063 & {[1.845]}
\end{array}
$$

Nous avons admis que les commissions et charges étaient une fonction proportionnelle de la prime pure (équations 8 et 9). La vérification empirique démontre qu'une telle relation simpliste n'est pas réaliste. Le fait de tenir compte des taux d'inflation présents et passés améliore considérablement la spécification de l'équation.

Dans le cas présent la structure de prix passés est de 7 années, ce qui n'est pas surprenant si l'on tient compte que les charges incluent les frais pour règlements de sinistres.

$\begin{array}{lcc}\text { montants } & \bar{R}^{2} & D . W . \\ \text { modèle de croissance linéaire } & 0.859 & 0.190 \\ \text { modèle autoregressif } & 0.997 & 1.125 \\ \text { modèle proposé } & 0.998 & 1.346 \\ (\mathrm{Co}+\mathrm{Ca})= & 0.0939 \bar{Q}_{t}+0.9295(\mathrm{Co}+\mathrm{Ca})_{t-1}+12.017 \text { PRIX } \\ \qquad[2.241] & {[9.400]} & {[1.038]}\end{array}$

Le problème de l'utilisation des lags d'Almon réside dans la difficulté d'espérer une structure stable ${ }^{10}$, ce qui est ennuyeux si l'on veut utiliser le modèle pour des simulations dans le futur. Afin de simplifier l'analyse, nous avons fait l'hypothèse d'une stabilité dans le temps de la distribution des coefficients sur les prix, et nous avons utilisé une transformation de Koyck en introduisant la valeur des commissions et charges à la période précédente.

S'il existe des économies d'échelle dans le temps, nous devrions trouver non pas une relation linéaire, mais une courbe. Toute fonction continue ayant une $k$ ième dérivée continue peut s'écrire sous la forme d'une série de Taylor, et nous avons donc testé la valeur élevée au carré de la variable " primes ». Le coefficient associé à cette variable s'est avéré positif, mais faiblement significatif pour l'assurance-dommage.

A nouveau, la relation incluant l'incidence de l'inflation est beaucoup plus satisfaisante selon le critère statistique que nous avons choisi (maximisation du couple $\bar{R}^{2}, D$. W.).

10 Voir à ce sujet J. J. Thomas [1978]. 
B) La sinistralité

Nous avons voulu à nouveau mesurer l'influence de la structure de prix présents et passés sur le ratio de sinistralité :

$\begin{array}{lcc} & \bar{R}^{2} & D . W . \\ \text { modèle de croissance linéaire } & 0.289 & 0.334 \\ \text { modèle autoregressif } & 0.782 & 1.325 \\ \text { modèle proposé } & 0.918 & 2.075 \\ & & \\ & & \end{array}$

polynôme du $3^{\text {e }}$ degré sans contraintes

\begin{tabular}{|c|c|c|}
\hline Lag 0 & -0.0051 & {$[-4.626]$} \\
\hline Lag 1 & -0.0032 & {$[-8.288]$} \\
\hline ag 2 & -0.0010 & {$[-2.448]$} \\
\hline $\mathrm{gg}$ & 0.0009 & {$\left[\begin{array}{ll}{[} & 2.160\end{array}\right]$} \\
\hline & 0.0027 & 7.318] \\
\hline $\mathrm{gg}$ & 0.0038 & [ 10.320$]$ \\
\hline & 0.0041 & {$\left[\begin{array}{ll}9 & 9.255\end{array}\right]$} \\
\hline & 0.0032 & $8.286]$ \\
\hline & 0.0009 & {$\left[\begin{array}{ll}2.27 \\
{[}\end{array}\right.$} \\
\hline
\end{tabular}

Comme l'on pouvait s'y attendre, la structure du polynôme de la variable inflation est semblable à celle décrite précédemment pour les charges d'exploitation. Par contre, il est intéressant de noter la pondération négative du début de période qui semble confirmer qu'en cas d'accélération de l'inflation, il y a accélération de la cadence de règlement de certains sinistres. En effet, lorsque la hausse des prix s'accélère, ce n'est pas seulement le montant des sinistres payés qui s'alourdit, c'est aussi celui de tous les sinistres en suspens. Il semble que l'accélération de l'inflation à partir des années 70 ait entraîné une modification du comportement des gestionnaires des compagnies. L'explication se confirme au regard du tableau des corrélations partielles entre prix et montant des sinistres réglés quand l'on considère trois sous-périodes dans les vingt-cinq dernières années.

Tableau 1 - Corrélations partielles entre prix et montant des sinistres réglés
période 1952-1959
0.177
période 1960-1967
0.332
période $1969-1975$
0.887 
C) Accroissement des provisions techniques

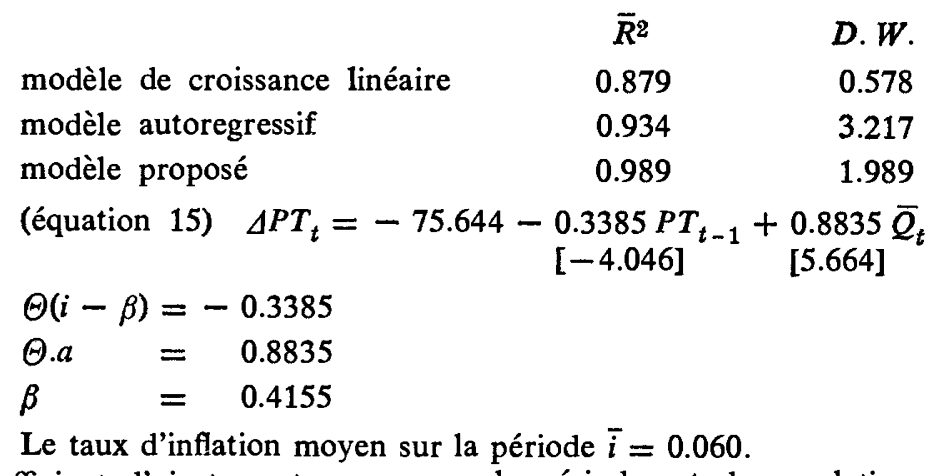

Le coefficient d'ajustement moyen sur la période est donc relativement proche de l'unité $\Theta=.9527$, et la valeur réelle du coefficient associé aux primes pures est lui aussi proche de l'unité $a=.9274$.

Ici, la formalisation du modèle découle de l'analyse conceptuelle. La conjoncture économique influence indirectement l'accroissement des provisions techniques par l'intermédiaire du chiffre d'affaires en primes pures réalisé chaque année.

\section{Evolution des opérations de l'assurance-dommages face à la conjoncture économique}

\subsection{Evolution des résultats}

Nous avons voulu observer dans cette dernière partie l'évolution des résultats du secteur des assurances-dommages. Le calcul du solde de souscription $W_{1}$ a été effectué à partir de la relation comptable suivante :

$$
W_{1}=Q-[C o+C a+S+\Delta P T]
$$

Le calcul de $W_{1}$ sur la période 1955-1976 montre que les opérations d'assurance se soldent par une perte d'exploitation. Nous avons comparé (Figure 2) l'inverse du ratio total d'exploitation (le rapport du total des frais de chargement et du coût de sinistralité aux primes encaissées) au rapport des pertes d'exploitation sur les primes encaissées (la différence s'expliquant principalement par l'accroissement des provisions techniques comme le montre la relation 16). La corrélation n'est pas parfaite et l'on observe depuis le début des années soixante un cycle de quatre ans affectant les pertes d'exploitation (à savoir 1963, 1967, 1971 et 1975).

Afin d'observer l'évolution du solde de souscription, il est donc primordial de tenir compte de l'accroissement des provisions techniques mais aussi du flux de primes encaissées $Q$. En ce qui concerne ce flux de primes, on sait qu'il n'évolue pas indépendamment de la conjoncture économique et nous avons décidé d'utiliser dans cette étude les résultats publiés récemment dans cette même revue ${ }^{11}$.

11 J.-François Outreville [1980]. 
Figure 2: Mouvement cyclique des pertes d'exploitation

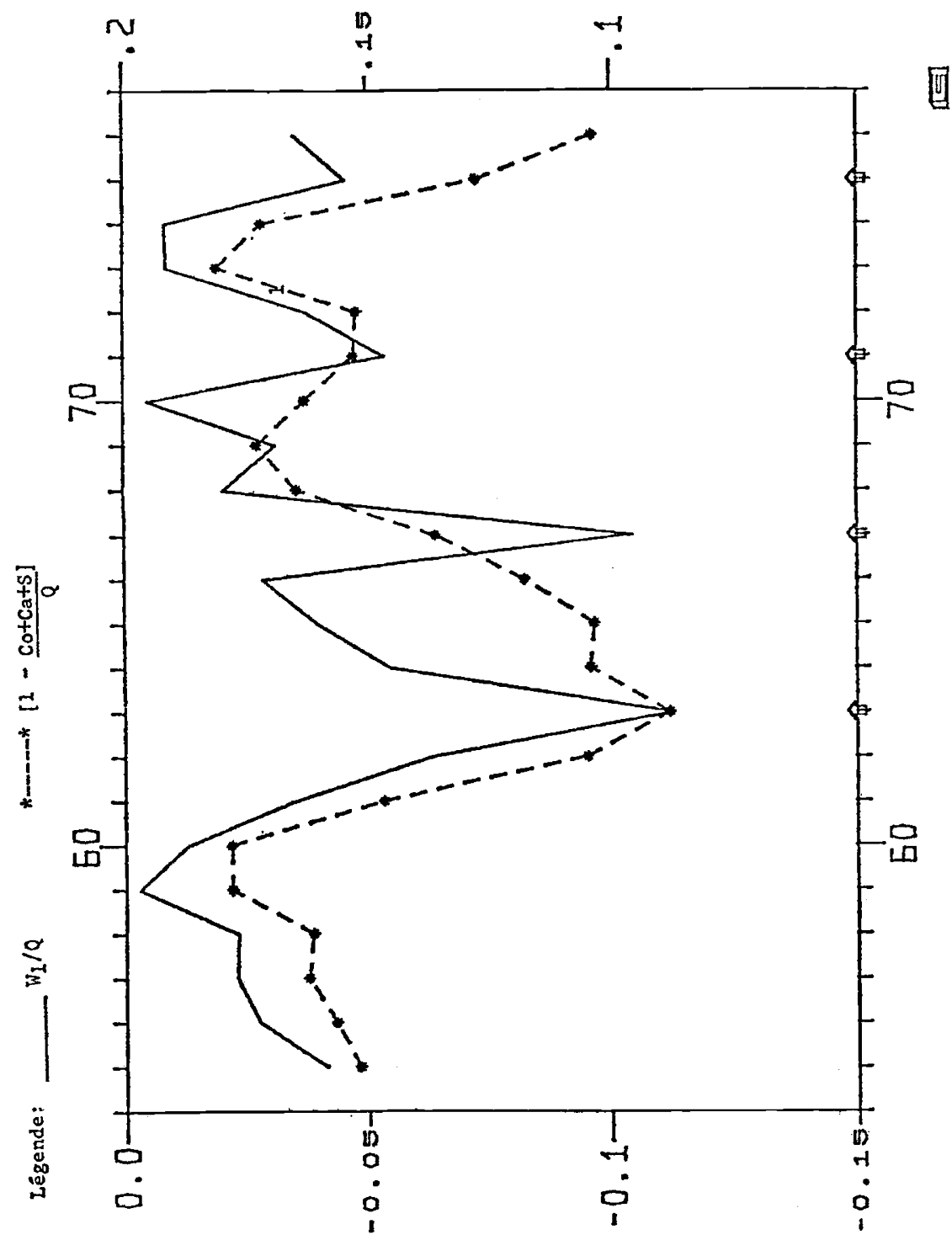




\subsection{Simulation d'hypothèses de conjoncture économique}

La prise en compte, dans certaines équations, des primes encaissées, posait le problème de l'estimation des paramètres dans le cadre d'équations simultanées. La méthode standard qui consiste à utiliser les doubles moindres carrés n'est pas directement applicable ici, aussi avons-nous eu recours à la méthode des variables intrumentales $\mathbf{1 2}$ en utilisant les valeurs estimées des primes obtenues par régression sur la période d'étude 1955-1975.

Afin de nous limiter à l'observation des effets de l'inflation sur la période de simulation 1976-1985, nous avons formulé deux hypothèses à partir de la situation de 1975 (taux d'inflation de $9.40 \%$ ) :

hypothèse 1 : taux d'inflation croissant $(14.5 \%$ en 1985$)$

hypothèse 2 : taux d'inflation décroissant ( $5 \%$ en 1985)

toutes choses étant égales par ailleurs, c'est-à-dire pour les variables macroéconomiques influençant les encaissements de primes, un même taux de chômage élevé et une hypothèse réaliste de faible croissance économique ${ }^{13}$.

Nous présentons (Figure 3) les résultats des simulations des hypothèses 1 et 2 pour le ratio $W_{1} / P T_{t-1}$ (rapport des pertes d'exploitation aux provisions techniques accumulées à la période précédente) qui correspond au rendement minimum des placements représentatifs de ces réserves afin que le solde global des opérations (à savoir $W_{2}$ ) soit nul.

Afin de connaître l'incidence d'une forte reprise de la croissance économique, nous avons formulé une troisième hypothèse (hypothèse 3 ) envisageant un retour à une croissance de $5.7 \%$ de 1980 à 1985.

Toutes choses étant égales par ailleurs, il apparaît nettement qu'un taux d'inflation élevé n'entraîne pas de détérioration des résultats. En fait, une forte croissance de l'économie semble être le moteur principal de bons résultats pour les compagnies d'assurance-dommages.

Les effets d'un taux d'inflation élevé sont complexes ; une comparaison graphique du rendement des placements des compagnies d'assurance-dommages avec le taux d'inflation montre (Figure 4) que pour les années 1969 puis 1974 à 1976, le rendement des placements ne suffit pas à conserver la valeur réelle des actifs. Si l'on reprend le schéma de détermination des provisions techniques et des placements, l'accroissement des capitaux propres (la richesse nette du secteur) est directement affecté ${ }^{14}$.

12 Voir à ce sujet Brundy and Jorgenson [1971].

13 Le modèle prenant en compte l'influence sur les primes de ces trois variables fondamentales: inflation, chômage et croissance, a été expliqué dans un article récent de J.-F. Outreville [1980].

14 En fait, d'un point de vue purement comptable, le total des placements des sociétés est calculé d'après la valeur au bilan et non pas d'après la valeur estimée aux cours de bourse. Le flux financier $F$ correspond donc plus à un rendement des placements qu'à une rentabilité des opérations financières tenant compte de l'appréciation (ou de la dépréciation) des actifs. 
Figure 3 : Simulation d'hypothèses de conjoncture économique et conséquences sur les pertes d'exploitation

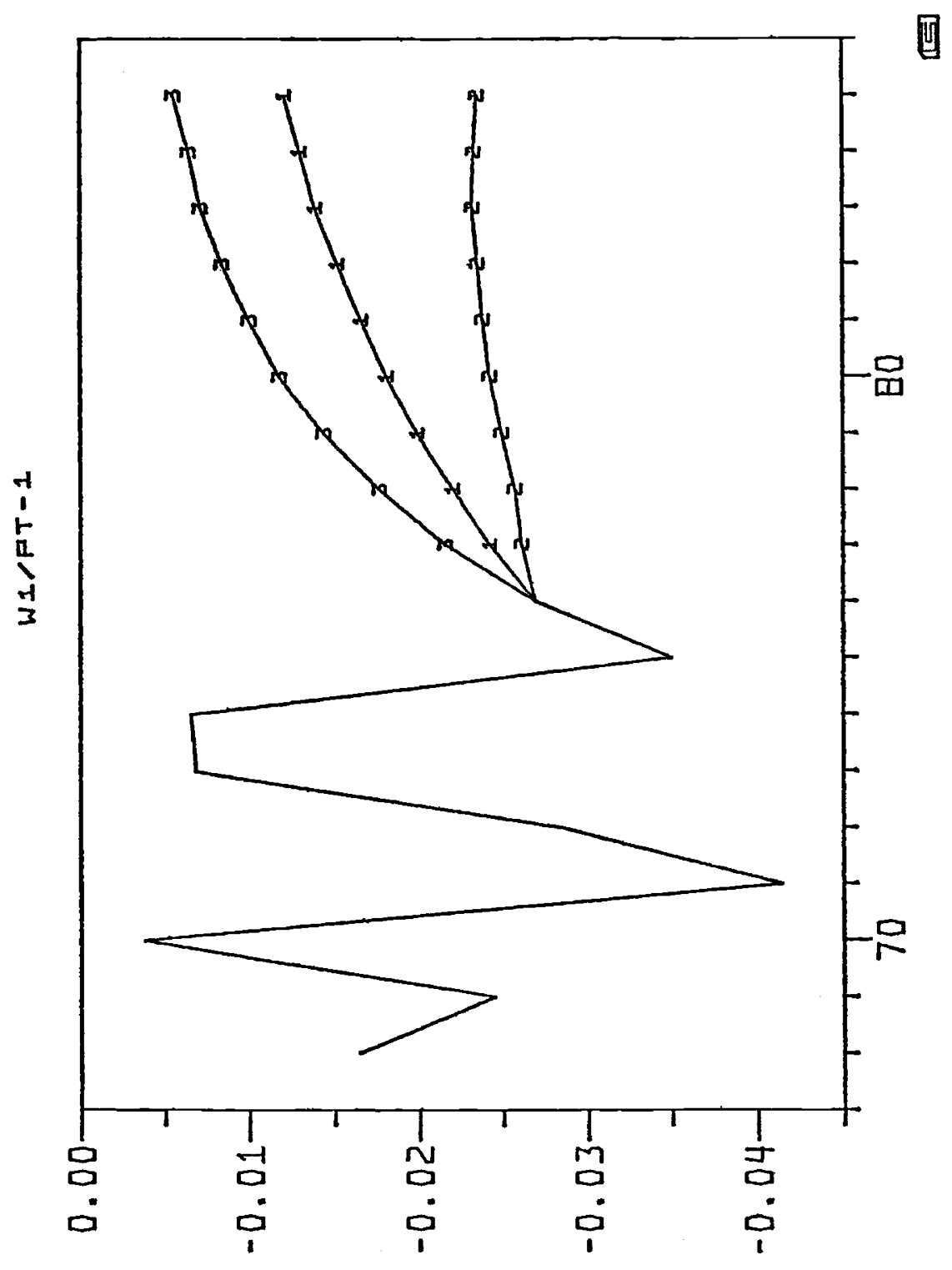


Figure 4 : Rentabilité comparée des placements des compagnies d'assurance et indice des prix

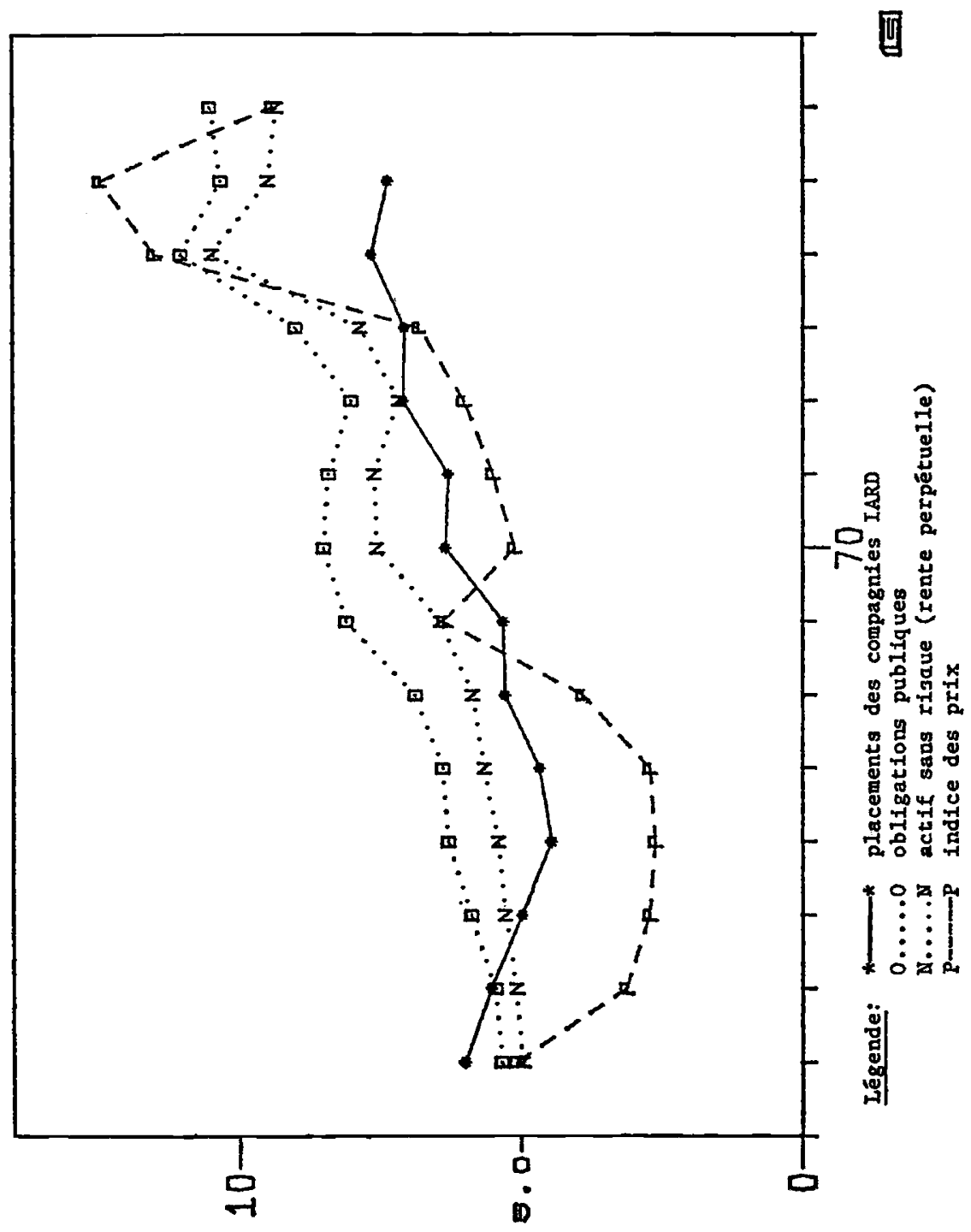


Il est évident qu'en ce qui concerne les résultats d'exploitation proprement dits, à savoir le flux $W_{2}$, le rendement des placements est suffisant pour réaliser l'égalité comptable (3) définie dans la seconde section. Par contre, il n'est pas évident qu'un taux d'inflation élevé permettra de réaliser un profit pur financier $W_{3}$ qui viendra accroître l'ensemble des fonds propres.

\section{Conclusion}

Nous venons de présenter la modélisation de l'ensemble des agrégats du schéma de détermination des provisions techniques et des placements, et le résultat de simulations d'hypothèses de conjoncture économique sur les résultats comptables des compagnies d'assurance I.A.R.D. en France. Dans ce modèle, l'accroissement des capitaux propres (la richesse nette du secteur) est considéré comme le résultat de l'identité comptable :

$$
\begin{aligned}
\text { (Primes }+ \text { flux financier }) & - \text { (commissions }+ \text { charges) } \\
& - \text { (paiement des sinistres et capitaux échus) } \\
& - \text { (accroissement des provisions techniques). }
\end{aligned}
$$

Cette représentation a été volontairement simplifiée, et l'on mentionnera un point important qui n'a pas été abordé, et qui mériterait une étude spécifique, à savoir, la prise en compte de l'impact de la réassurance.

En outre, une analyse complète des éléments comptables devrait étudier la politique des dividendes (ou participation), les impôts et taxes, les dettes et créances à court terme.

Dans la troisième section de cette étude, nous avons démontré qu'il était critiquable d'envisager des relations trop simplistes quant aux opérations des compagnies d'assurance-dommages. D'autre part, dans la mesure où les variables du modèle sont directement affectées par le taux d'inflation et le taux de rendement des actifs financiers, il est difficile de conclure que l'assurance se développe indépendamment de l'évolution économique en général ${ }^{15}$.

Bien que l'on considère généralement que les intermédiaires financiers s'adaptent assez rapidement aux situations nouvelles, il en est, croissance faible et inflation élevée, qui assombrissent fortement les résultats actuels et les perspectives futures. Les compagnies d'assurance n'échappent pas à cette règle.

Nous avons simulé, dans la dernière section de cette étude, trois scénarios de conjoncture économique sur les résultats d'exploitation du secteur. Même si les tendances observées ne sont qu'une simplification de la réalité, elles démontrent clairement notre point de vue.

15 C'est à cette conclusion qu'en arrivait une étude publiée par le CAPA en 1976. 


\section{Annexe : Ratios statistiques}

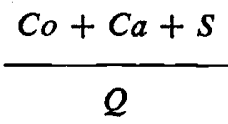

1976

1975

1974

1973

1972

1971

1970

1969

1968

1967

1966

1965

1964

1963

1962

1961

1960

1959

1958

1957

1956

1955
89.62

87.25

82.84

81.93

84.79

84.74

83.73

82.75

83.56

86.39

88.22

89.66

89.59

91.21

89.53

85.34

82.19

82.20

83.87

83.78

84.34

84.83

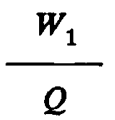

$-0.0354$

$-0.0457$

$-0.0086$

$-0.0089$

$-0.0375$

$-0.0537$

$-0.0050$

$-0.0313$

$-0.0205$

$-0.1042$

$-0.0286$

$-0.0400$

$-0.0548$

$-0.1127$

$-0.0633$

$-0.0352$

$-0.0129$

$-0.0029$

$-0.0231$

$-0.0230$

$-0.0276$

$-0.0414$

$$
\frac{W_{1}}{P T_{t-1}}
$$

$-0.0269$

$-0.0350$

$-0.0065$

$-0.0068$

$-0.0285$

$-0.1014$

$-0.0038$

$-0.0245$

$-0.0165$

$-0.0914$

$-0.0232$

$-0.0326$

$-0.0450$

$-0.0929$

$-0.0515$

$-0.0308$

$-0.0118$

$-0.0028$

$-0.0241$

$-0.0234$

$-0.0287$

$-0.0534$

Sources: Jean-François Outreville [1979]. 


\section{REFERENCES}

BALL, R., and BROWN, P. [1968] : An empirical evaluation of accounting income numbers », Journal of Accounting Research, 6 (Autumn 1968), 159-178.

BALL, R., and WATTS, R. [1972]: \&ome time series properties of accounting income numbers », Journal of Finance, 38 (June 1972), 663-681.

BEAVER, W. [1970]: « The time series behavior of earnings », in Empirical Research in Accounting : Selected Studies, Supplement to the Journal of Accounting Research, vol 8, 62-99.

BOHMAN, H. [1979] : « A mathematical model of insurance business and how it may be used », Geneva Papers on Risk and Insurance, 11 (January 1979), 34-39.

BORCH, K. [1966] : « Control of a portfolio of insurance contracts », Astin Bulletin, 4 (1966), 59-71.

BORCH, K. [1967] : * Dynamic decision problems in an insurance company », Astin Bulletin, 5 (1967), 118-131.

BORCH, K. [1974]: The Mathematical Theory of Insurance, Lexington Books, Lexington (Mass.).

BROOKS, L. D., and BUCKMASTER, D. A. [1976] : \& Further evidence on the time series properties of accounting income », Journal of Finance, 31 (December 1976), 1359-1373.

BROWN, P., and BALL, R. [1967] : \& Some preliminary findings on the association between the earnings of a firm, its industry and the economy $»$, in Empirical Research in Accounting : Selected Studies, Supplement to the Journal of Accounting Research, vol. 5, 55-57.

BRUNDY, J.M., and JORGENSON, D.W. [1971] : «Efficient estimation of simultaneous equations by instrumental variables », Review of Economics and Statistics, 53 (August 1971), 207-224.

CAPA [1976] : L'Assurance et son Environnement économique, no 192, Paris.

CUMMINS, J. D. [1975] : An Econometric Model of the Life Insurance Sector in the U.S., Lexington Books, Lexington (Mass.).

DE FINETTI, B. [1957]: «Su una impostazione alternative della theoria collectiva del rischio », International Congress of Actuaries, vol. 2, 433-443 (cité dans BORCH [1966] et [1967]).

DEPOID, P. [1967] : Applications de la Statistique aux Assurances, Berger-Levrault, Paris.

LOOKABILL, L. [1970]: "Some additional evidence on the time series properties of accounting earnings », Accounting Review, 51 (October 1970), 724-738.

LUNDBERG, F. [1909] : «Zur Theorie der Rückversicherung 》, Transactions of the International Congress of Actuaries.

OUTREVILLE, J.-F. [1979]: Un Modèle Macroéconomique du Secteur de l'Assurance en France, Thèse de Doctorat d'Etat, Orléans.

OUTREVILLE, J.-F. [1980]: «Dépenses d'assurances, primes encaissées: une approche macroéconomique », Geneva Papers on Risk and Insurance, 17 (juin 1980), 23-44.

THOMAS, J. J. [1978] : « Some problems in the use of Almon's technique in the estimation of distributed lags », Empirical Economics, 2 (3/1978), 175-193. 